\title{
Simultaneous broadband microwave downconversion and programmable complex filtering by optical frequency comb shaping
}

\author{
Victor Torres-Company, ${ }^{1,2, *}$ Daniel E. Leaird, ${ }^{1}$ and Andrew M. Weiner ${ }^{1}$ \\ ${ }^{1}$ School of Electrical and Computer Engineering, Purdue University, 465 Northwestern Avenue, West Lafayette, Indiana 47907, USA \\ ${ }^{2}$ Departament de Física, Universitat Jaume I, Castelló de la Plana, Castelló 12081, Spain \\ ${ }^{*}$ Corresponding author: torres31@purdue.edu
}

Received June 7, 2012; revised August 8, 2012; accepted August 16, 2012;

posted August 17, 2012 (Doc. ID 170030); published September 20, 2012

\begin{abstract}
High-repetition-rate optical frequency combs can act as broadband photonic mixers and downconvert a microwave signal to an intermediate frequency (IF) band so that it becomes accessible with high-speed electronics. In this Letter, we show that with line-by-line pulse shaping and dispersive propagation, the photonic mixer can simultaneously perform programmable multitap complex-coefficient-filtering within the IF band. This solution opens new possibilities for microwave signal processing by combining the flexibility of optoelectronic frequency comb technology with high-speed analog-to-digital converters. (c) 2012 Optical Society of America

OCIS codes: $\quad 060.5625,070.2615,320.5540$.
\end{abstract}

One of the most researched applications in the field of radio-frequency $(\mathrm{RF})$ photonics is the realization of programmable filtering of microwave signals [1]. Photonics approaches bring features difficult or impossible to attain by purely electronic solutions, such as simultaneous tuning and reconfigurability, high processing speed, broadbandwidth operation, or compatibility with fiber optics [2]. Recently, optoelectronic frequency comb generators [3] have emerged as a convenient platform for microwave photonic (MWP) filtering because of their high repetition rate, large number of lines, and the possibility to tune the central wavelength and timing independently of the repetition rate [4]. Thanks to these characteristics, MWP filters with high side-lobe suppression or submicrosecond tuning speeds have been reported [5]. When combined with line-by-line pulse shaping, reconfigurable filtering and programmable phase control is also possible [6]. These features are beyond the state of the art in electronics and illustrate very well the potential of MWPs.

An alternative to process microwave signals consists of shifting their spectral content to an intermediate frequency (IF) so that they can be conveniently handled by a high-speed analog-to-digital converter (ADC). Several optical techniques for microwave downconversion have been reported, mainly involving electro-optic mixing $[\underline{7}, \underline{8}]$, optical heterodyning $[\underline{9}, \underline{10}]$, or high-speed sampling [11]. The first two methods can be combined with narrowband optical filtering to achieve simultaneous downconversion and analog filtering before the detection stage $[8,10]$. In these techniques, however, it is difficult to implement programmable filtering, because the relatively narrow IF bandwidth with respect to the optical wavelength calls for a high-quality, fine-resolution reconfigurable optical filter. Alternatively, the high-speed sampling approach involves the use of a high-repetition-rate optical pulse train to sample the microwave signal in the temporal domain and then to perform photodetection of the modulated pulses [11]. In this method, the frequency content of the microwave signal can be as large as half the train's repetition rate. Optoelectronic frequency combs with repetition rates $\sim 10-40 \mathrm{GHz}$ provide an ideal platform to perform this task [12] because they ensure an IF bandwidth compatible with state-of-the-art ADCs, are phase stable, and have the potential to achieve low timing jitter [13]. In this Letter, for the first time to our knowledge, we show that downconversion sampling and programmable MWP filtering can be accomplished simultaneously by combining optoelectronic frequency comb technology with line-by-line pulse shaping. The key enabling point is the use of an MWP dispersive delay-line configuration [1]. This solution allows for high-quality programmable filtering in the IF band with large timebandwidth product and relatively long ( $>1 \mathrm{~ns}$ ) temporal apertures.

Our setup is depicted in Fig. 1(a). A frequency comb with $N$ lines is used as multiwavelength light source and programmed in a line-by-line manner with the aid of a pulse shaper. The light is then modulated in a singlesideband (SSB) modulator driven by the microwave signal to be filtered and downconverted. The signal's frequency content must lie within one of the Nyquist zones illustrated in Fig. 1(b) [4]. We note that the flexibility in choosing the repetition rate in optoelectronic frequency combs enables the user to accommodate these zones to their needs. Let us consider a microwave signal, $f$, higher than the repetition rate of the comb source, $f_{r}$,

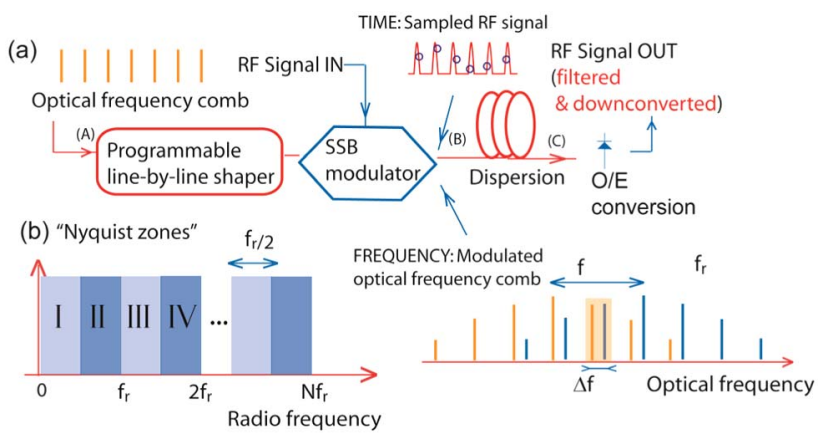

Fig. 1. (Color online) (a) Setup to perform downconversion and complex-coefficient filtering. (b) The input microwave signal must lie in one of the shadowed regions. 
that is, $f=L f_{r}+\Delta f$, with $L$ being an integer number and $\Delta f$ being the offset with respect to the nearest adjacent comb sideband $[L=2$ in Fig. 1(a) for illustration purposes]. By placing a dispersive element after modulation, the setup is transformed into a dispersive multitap MWP downconversion filter. In consequence, the role of the dispersive element is to introduce a constant temporal delay between consecutive optical carriers [1]. In this way, upon detection by a photodiode (PD) with cutoff frequency $f_{r} / 2$, the output electrical signal is composed by a summation of beat terms at frequency $\Delta f$. Mathematically, it can be shown that the process is described by the complex linear transfer function

$$
H(\Delta f)=H_{\text {mod }}(\Delta f) \sum_{m} A_{m} \exp (i m 2 \pi \Delta f \tau)
$$

with

$$
A_{m}=E_{L+m}^{*} E_{m}
$$

Here, $\tau=2 \pi \Phi_{2} f_{r}$ is the tap delay, with $\Phi_{2}$ the group delay dispersion amount (in $\mathrm{ps}^{2}$ ) and $A_{m}$ the complex tap coefficients, related to the complex field of the shaped $m$ th comb line, $E_{m}$, before modulation. The first factor on the right-hand side of Eq. (1a) accounts for a dispersion-induced phase factor, $H_{\text {mod }}(\Delta f) \propto \exp \left[i \Phi_{2}\right.$ $\left.(2 \pi \Delta f)^{2} / 2\right] \exp \left[i L 4 \pi^{2} \Phi_{2} f_{r} \Delta f\right]$.

Equation (1) is a key result of this Letter. It establishes a linear relation between the input $f$ and the output frequency $\Delta f$. The second term on the right-hand side of Eq. (1) indicates that it is possible to perform programmable complex filtering within the IF band through the proper configuration of the coefficients $E_{m}$ with the pulse shaper. This highlights the need to keep a stable phase relation across the optical bandwidth of the source. This situation is in radical contrast to multitap MWP filtering without downconversion [4], for which the role of the spectral phase of the comb is irrelevant.

The optoelectronic frequency comb generator is generated by placing in tandem an intensity (EOM) and phase modulator (EOPM), both driven by a $10 \mathrm{GHz}$ oscillator. The signal going into the EOPM is amplified by a high-power RF amplifier. When the DC bias and RF delay are properly set, the comb provides 20 lines at $-10 \mathrm{~dB}$ bandwidth. Thus, this comb has the capability to downconvert a microwave signal at a frequency carrier as high as $200 \mathrm{GHz}$ having up to $5 \mathrm{GHz}$ instantaneous bandwidth. The limit on the maximum frequency carrier will be restricted by the bandwidth of the SSB modulator $(12 \mathrm{GHz}$ in our case). A single-mode-fiber (SMF) of $1.4 \mathrm{~km}$ is used to compensate for the spectral phase of the comb and get a compressed pulse with $\sim 5$ ps at FWHM as measured by an intensity autocorrelation apparatus. The SSB modulator is a dual-drive Mach-Zehnder modulator biased at the quadrature point and driven by a pair of copies with 90 deg phase difference of the microwave signal to be processed. To illustrate the capabilities of the comb for downconversion, we send an $11 \mathrm{GHz}$ single-frequency signal from an RF synthesizer to the input of the modulator. The modulated light is detected by a $22 \mathrm{GHz}$ PD and monitored with an RF spectrum analyzer. The RF spectrum is shown in Fig. 2(a). As can be seen, the $11 \mathrm{GHz}$ tone is downconverted to $1 \mathrm{GHz}$ in the $0-5 \mathrm{GHz}$ IF band. The beat with the second-nearest sideband of the $10 \mathrm{GHz}$ comb also leads to downconversion at $9 \mathrm{GHz}$, as expected. To check the precision of the IF, we sweep the RF synthesizer from $10-5 \mathrm{GHz}$ and monitor the frequency position of the downconverted signal in the $0-5 \mathrm{GHz} \mathrm{IF}$ band. As shown in Fig. 2(b), there is an excellent agreement between the measured and expected IF, taking into account the oscillator frequency of the comb source (the deviation is within the $100 \mathrm{~Hz}$ instrument resolution). Next, to show that multiple RFs can be simultaneously downconverted, we launch a microwave signal linearly chirped from 10.1-11.1 GHz over a window of $10.4 \mathrm{~ns}$, synthesized by an electrical arbitrary waveform generator with 24 GS /s sampling rate. A single-shot acquisition of the input signal captured by a real-time oscilloscope working at $50 \mathrm{GS} / \mathrm{s}$ is displayed in Fig. 2(c), and the calculated RF spectrum is shown in Fig. 2(d). The output downconverted signal at point $B$ in Fig. 1 , after photodetection, $4 \mathrm{GHz}$ low-pass filtering, and amplification is shown in Fig. 2(e), together with the corresponding RF spectrum shown in Fig. 2(f). The whole bandwidth content is downconverted to the expected region while preserving the chirp rate.

To show the capability of the system to perform reconfigurable complex analog filtering within the IF band, we introduce between the comb and the SSB modulator a pulse shaper (Finisar WaveShaper 1000S), which enables amplitude and phase control over the $C$-band with $10 \mathrm{GHz}$ resolution. At the output of the shaper, the signal is amplified with an erbium-doped fiber amplifier (EDFA) (not shown in Fig. 1). After modulation, we send the signal to a dispersion compensating fiber (DCF) module
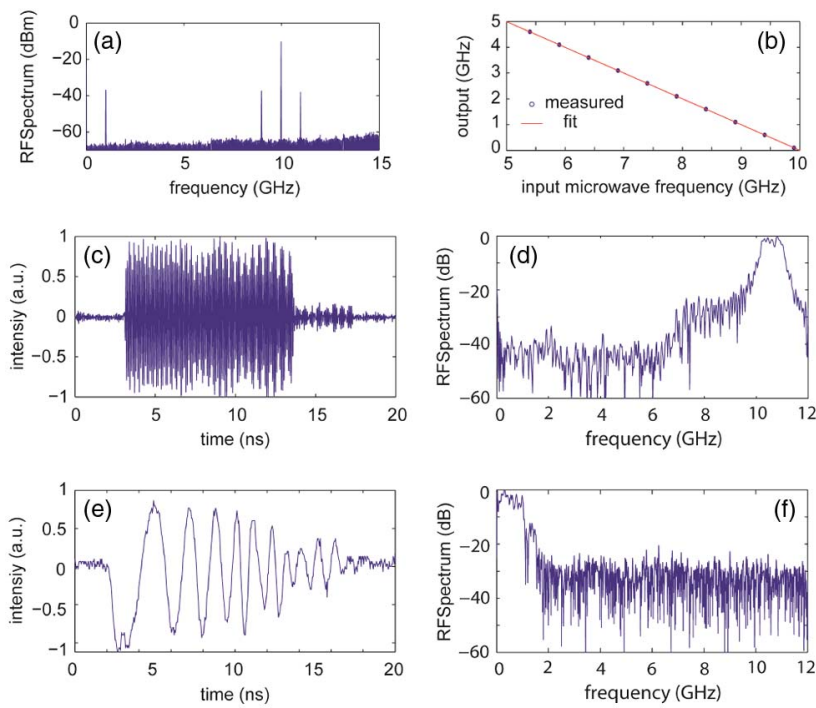

Fig. 2. (Color online) Microwave downconversion using an optical frequency comb. (a) RF spectrum measured at point (B) in Fig. 1 ( $1 \mathrm{MHz}$ RBW) considering an $11 \mathrm{GHz}$ microwave signal as input to the SSB modulator. (b) Measured IF when the input signal is swept from 5-10 GHz. Characteristics of another broadband signal to be downconverted: (c) temporal domain and (d) frequency domain. (e) Single-shot trace of the downconverted signal from (c), and (f) RF spectrum. 

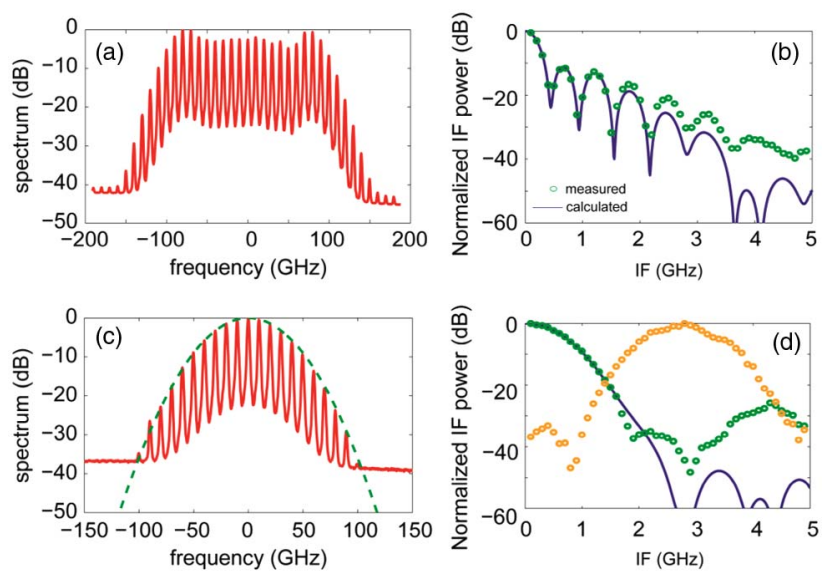

Fig. 3. (Color online) (a) Unapodized optical frequency comb. (b) Resulting IF normalized power distribution (circles) and expected (solid curve). (c) Apodized frequency comb (solid curve) and target envelope (dashed line). (d) Resulting IF normalized power distribution (green circles) and expected (orange circles). When dispersion is introduced before the SSB modulator, the IF filter response can be tuned (open circles).

that introduces a tap delay of $96 \mathrm{ps}$. In the first example, the pulse shaper acts as an all-pass device, that is, no mask is synthesized. The resulting comb is shown in Fig. 3(a). We repeat the same procedure as before by sweeping an RF signal in the $5-10 \mathrm{GHz}$ range and monitoring the $\mathrm{RF}$ power of the corresponding downconverted frequency. The normalized power distribution is shown in circles in Fig. 3(b). The power distribution matches the corresponding power from Eq. (1), taking into account the measured optical spectrum. It is clear that the dispersive fiber link acts as an MWP filter within the IF band. The shape of this low-pass filter can be easily reconfigured by programming the comb shape. To show this, we configure the pulse shaper to get a frequency comb with a Gaussian envelope, as shown in Fig. 3(c), in which the target envelope is displayed with a dashed curve. We sweep again the input $\mathrm{RF}$ and observe that the normalized power distribution within the IF band changes accordingly, as expected from Eq. (1). In addition to amplitude filtering, Eq. (1) predicts programmable phase filtering. This opens up the possibility to achieve resonance tuning through the IF band. Recalling that $A_{m}=E_{L+m}^{*} E_{m}$, this can be achieved by introducing a quadratic phase factor on the optical taps, that is, $E_{m}=$ $E_{m}^{\prime} \exp \left(i 2 \pi C m^{2}\right)$, with $C$ being a real-valuated constant. In this way, $A_{m}=\exp \left(-i 2 \pi C L^{2}\right) A_{m}^{\prime} \exp (-i 4 \pi C L m)$, with $A_{m}^{\prime}=E_{L+m}^{*} E_{m}^{\prime}$. The filter transfer function is then modified to $H^{\prime}(\Delta f)=H_{\text {mod }}(\Delta f) \sum A_{m}^{\prime} \exp [i m 2 \pi \tau(\Delta f-$ $\left.f_{0}\right)$ ], where an irrelevant quadratic phase factor has been omitted for clarity. The new central frequency is $f_{o}=2 C L / \tau$. To show the tuning capabilities of the system, we place a dispersive fiber link of $20.5 \mathrm{~km}$ after the shaper. This introduces the required quadratic phase onto the complex coefficients to shift the resonance band to $3 \mathrm{GHz}$, as shown in Fig. 3(d). The need to introduce spectral quadratic phase (i.e., dispersion) to shift the resonance bandpass is due to the phase difference operation implicit in the tap coefficients. This is in contrast to the tuning mechanisms in other comb-based configuration that do not perform downconversion sampling [므무] , where tuning may be achieved in an interferometric configuration.

In summary, we have shown that optical frequency comb technology, when combined with line-by-line pulse shaping and dispersive propagation, provides a convenient platform to perform simultaneously programmable complex filtering and downconversion sampling of microwave signals. With a $10 \mathrm{GHz}$ optoelectronic frequency comb, we have shown tunable and reconfigurable filtering within the $0-5 \mathrm{GHz}$ IF band.

This project was supported in part by the Naval Postgraduate School under grant N00244-09-1-0068 under the National Security Science and Engineering Faculty Fellowship program and grant ECCS-1102110 from the National Science Foundation. Any opinion, findings, and conclusions or recommendations expressed in this publication are those of the authors and do not necessarily reflect the views of the sponsors. Victor TorresCompany gratefully acknowledges funding from a Marie Curie Fellowship (PIOF-2009-234996).

\section{References}

1. J. Capmany, B. Ortega, and D. Pastor, J. Lightwave Technol. 24, 201 (2006).

2. J. Capmany and D. Novak, Nat. Photon. 1, 319 (2007).

3. H. Murata, A. Morimoto, T. Kobayashi, and S. Yamamoto, IEEE J. Sel. Top. Quantum Electron. 6, 1325 (2000).

4. E. Hamidi, D. E. Leaird, and A. M. Weiner, IEEE Trans. Microwave Theor. Tech. 58, 3269 (2010).

5. V. R. Supradeepa, C. M. Long, R. Wu, F. Ferdous, E. Hamidi, D. E. Leaird, and A. M. Weiner, Nat. Photon. 6, 186 (2012).

6. M. H. Song, V. Torres-Company, A. J. Metcalf, and A. M. Weiner, Opt. Lett. 37, 845 (2012).

7. G. K. Gopalakrishnan, W. K. Burns, and C. H. Bulner, IEEE Trans. Microwave Theor. Tech. 41, 2383 (1993).

8. V. R. Pagan, B. M. Haas, and T. E. Murphy, Opt. Express 19, 883 (2011).

9. A. K. M. Lam, M. Fairburn, and A. F. Jaefer, IEEE Trans. Microwave Theor. Tech. 54, 240 (2006).

10. K.-Y. Tu, M. S. Rasras, D. M. Gill, S. S. Patel, Y.-K. Chen, A. E. White, A. Pomerene, D. Carothers, J. Beattie, M. Beals, J. Michel, and L. C. Kimerling, J. Lightwave Technol. 28, 3019 (2010).

11. P. W. Juodawlkis, J. J. Hargreaves, R. D. Younger, G. W. Titi, and J. C. Twichell, J. Lightwave Technol. 21, 3116 (2003).

12. Y. Zhao, X. Pang, L. Deng, X. Yu, X. Zheng, and I. T. Monroy, IEEE Photon. Technol. Lett. 24, 16 (2012).

13. S. Xiao, L. Hollberg, N. R. Newbury, and S. A. Diddams, Opt. Express 16, 8498 (2008). 